\title{
Índices vegetais fotogramétricos no milho em sucessão a plantas de cobertura do solo em Areia, Paraíba (Brasil)
}

Photogrammetric plant indices in corn in succession to ground cover crops in Areia, Paraíba

(Brazil)

Índices fotogramétricos de plantas en maíz en sucesión a cultivos de cobertura del suelo en Areia, Paraíba (Brasil)

\begin{abstract}
Resumo
Para melhor controle e eficiência dos cultivos agrícolas nas regiões semiáridas brasileiras, o uso de avaliações com dados provenientes de sensoriamento remoto em conjunto com sistemas de informações geográficas (SIG) estão sendo amplamente utilizados. Neste trabalho objetivou-se comparar os índices vegetativos em sistema de cultivo de milho e plantas de cobertura do solo nas condições semiáridas do Brejo da Paraíba. A pesquisa foi realizada em Areia, Paraíba, Brasil, nos anos agrícolas de 2018/2019 e 2019/2020. O delineamento foi em blocos casualizados, com quatro repetições, no esquema fatorial $3 \times 6$, correspondente a três genótipos de milho (cultivar Robusto, milho crioulo Pontinha e híbrido AG1051) e seis sistemas de cultivo de plantas de cobertura [(Brachiaria ruziziensis, milheto (Pennisetum glaucum), feijão guandu (Cajanus cajan), Crotalária espectabilis, Crotalária juncea e como testemunha apenas o solo descoberto)], totalizando 18 tratamentos. Foram avaliados os índices vegetais de Visible Atmospherically Resistant Index, Redness Index, Normalized green-Red Difference Index, Ground Level Image Analysis, Excess Red-Green, Excess Red Vegetative Index, Excess Green Index e o Color Index of Vegetation Extraction. De maneira geral, os resultados demonstraram que para observar diferenças entre os genótipos de milho é indicado o uso do índice Excess Red Vegetative Index. Para observar diferenças do efeito das plantas de cobertura no milho, indica-se o Normalized green-Red Difference.
\end{abstract}

Palavras-chave: Plantas de cobertura; Zea mays; Drone; Sensoriamento remoto.

\begin{abstract}
For better control and efficiency of agricultural crops in Brazilian semiarid regions, the use of evaluations with data from remote sensing in conjunction with geographic information systems (GIS) are being widely used. This work aimed to evaluate the response of corn genotypes in a succession cultivation system to vegetation cover plants. The survey was conducted in Areia, Paraíba, Brazil, in the agricultural years of 2018/2019 and 2019/2020. The design was in completely randomized blocks, with four replications, in a $3 \times 6$ factorial scheme, corresponding to three corn genotypes (Robusto, Pontinha and AG1051) and six cultivation systems [(Brachiaria ruziziensis, millet (Pennisetum
\end{abstract}


glaucum), pigeonpea (Cajanus cajan), Crotalária espectabilis, Crotalária juncea and as witness only the uncovered soil)], totaling 18 treatments. Vegetable indices of Visible Atmospherically Resistant Index, Redness Index, Normalized green-Red Difference Index, Ground Level Image Analysis, Excess Red-Green, Excess Red Vegetative Index, Excess Green Index and the Color Index of Vegetation Extraction were evaluated. The results showed that to observe differences between the corn genotypes, the use of the Excess Red Vegetative Index is indicated. To observe differences in the effect of cover crops on corn, the Normalized green-Red Difference is indicated.

Keywords: Coverage plants; Zea mays; Drone; Remote sensing.

\section{Resumen}

Para un mejor control y eficiencia de los cultivos agrícolas en las regiones semiáridas brasileñas, se está utilizando ampliamente el uso de evaluaciones con datos de teledetección en conjunto con sistemas de información geográfica (SIG). Este trabajo tuvo como objetivo evaluar la respuesta de genotipos de maíz en un sistema de cultivo de sucesión a plantas de cobertura vegetal. La encuesta se realizó en Areia, Paraíba, Brasil, en los años agrícolas 2018/2019 y 2019/2020. El diseño fue en bloques completamente al azar, con cuatro repeticiones, en un esquema factorial $3 \times 6$, correspondientes a tres genotipos de maíz (Robusto, Pontinha y AG1051) y seis sistemas de cultivo [(Brachiaria ruziziensis, mijo (Pennisetum glaucum), guandú (Cajanus cajan), Crotalária espectabilis, Crotalária juncea y como testimonio solo el suelo descubierto)], totalizando 18 tratamientos. Se evaluaron los índices vegetales de Visible Atmospherically Resistant Index, Redness Index, Normalized green-Red Difference Index, Ground Level Image Analysis, Excess Red-Green, Excess Red Vegetative Index, Excess Green Index y Color Index of Vegetation Extraction. Los resultados mostraron que para observar diferencias entre los genotipos de maíz se indica el uso del Excess Red Vegetative Index. Para observar las diferencias en el efecto de los cultivos de cobertura sobre el maíz, se indica la Normalized green-Red Difference.

Palabras clave: Plantas de cobertura; Zea mays; Drones; Teledetección.

\section{Introdução}

A importância econômica do milho (Zea mays L.) pode ser caracterizado pelas diversas formas de utilização, como na alimentação humana e animal (Mumbach et al., 2017), como também pela utilização da silagem do milho, a qual é um importante componente para a pecuária em regiões semiáridas, devido a produção de massa verde associada ao teor de carboidratos solúveis, servindo para alimentação animal de corte e leiteiro (Pereira et al., 2012).

No entanto, um dos problemas dos cultivos comerciais é conseguir informações que possibilitem uma melhor tomada de decisão e redução de custos operacionais. Para isso, uma alternativa tem sido a adoção de novas tecnologias como o monitoramento dos cultivos através do uso de dados provenientes de sensores remotos, auxiliados por Sistemas de Informações Geográficas (SIG) (Kalisch et al., 2020; Ribeiro et al., 2021). Estes sistemas servem para o mapeamento, caracterização, estudo da evolução do uso do solo, gerenciamento de propriedades, dentre outras atividades ambientais e agronômicas, pois fornecem informações periódicas de extensas áreas e com menor custo do que as práticas tradicionais (Backes, 2010).

Neste sentido, com o suporte destas tecnologias, a utilização de uma variedade de índices de vegetação é desenvolvida para auxiliar no monitoramento da vegetação, onde a maior parte está baseada em interações entre a vegetação e a energia eletromagnética nos comprimentos de onda do vermelho e do infravermelho próximo (Acco, 2004).

Com base nessas informações, neste trabalho objetivou-se comparar os índices vegetativos em sistemas de cultivo de milho e plantas de cobertura do solo em condições semiáridas do Brejo da Paraíba.

\section{Metodologia}

Este trabalho trata-se de uma pesquisa quantitativa e experimental (Gerhardt \& Silveira, 2009).

\subsection{Local de estudo}

A pesquisa foi realizada na Fazenda Experimental Chã de Jardim ( $06^{\circ} 57^{\prime} 46^{\prime \prime} \mathrm{S}$ e $35^{\circ} 41^{\prime} 31^{\prime}$ ' O e altitude de $\left.600 \mathrm{~m}\right)$, no Centro de Ciências Agrárias da Universidade Federal da Paraíba, no município de Areia, Paraíba, Brasil, nos anos agrícolas 2018/2019 e 2019/2020. 
O solo da área experimental de natureza argilo-arenosa está classificado como Latossolo (Embrapa, 2006). O clima local de acordo com a classificação de Köppen é do tipo Aw’, quente e úmido (Alvares et al., 2013), com a temperatura média anual de $22{ }^{\circ} \mathrm{C}$, alta umidade e precipitação pluvial média anual de $1.400 \mathrm{~mm}$, sendo o quadrimestre mais chuvoso os meses de abril a julho (Ribeiro et al., 2018). A precipitação pluvial mensal durante o período experimental está apresentada na Figura 1.

Figura 1. Médias mensais de precipitação pluvial no local experimental.

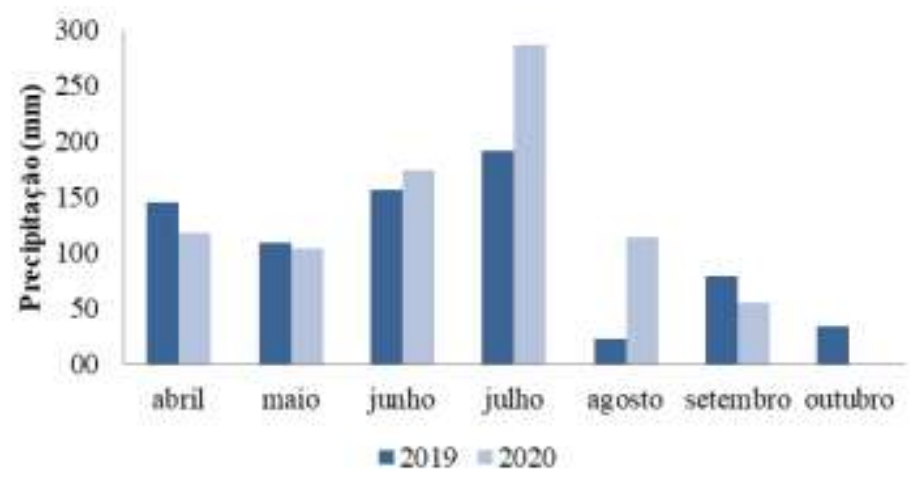

Fonte: Autores.

\subsection{Delineamento experimental}

O delineamento utilizado foi de blocos casualizados (DBC), com quatro repetições, no esquema fatorial $3 \times 6$, sendo três genótipos de milho (cultivar Robusto, variedade de milho crioulo (Pontinha) e um híbrido simples de milho (AG 1051) adaptados a região $\times$ seis sistemas de cultivo com plantas de cobertura vegetal do solo (Brachiaria ruziziensis, milheto (Pennisetum glaucum), feijão guandu (Cajanus cajan), Crotalária espectabilis, Crotalária juncea e a testemunha com solo descoberto)], totalizando 18 tratamentos.

\subsection{Manejo agronômico}

Foi realizado o preparo inicial do solo e a adubação de acordo com as recomendações da análise de solo. Cada parcela media 3,0 $\mathrm{m} \times 4,5 \mathrm{~m}$. As parcelas foram constituídas por nove fileiras de milho com 3,0 metros de comprimento e espaçamento entre fileiras de $0,5 \mathrm{~m}$, considerando como bordadura as três linhas laterais e $0,5 \mathrm{~m}$ das extremidades de cada linha de plantas.

No período da entressafra do milho 2018/2019 e 2019/2020 as plantas de cobertura foram semeadas nas parcelas, em 15 de abril de cada ano. A densidade de plantio e população final de plantas estão apresentadas na Tabela 1 . Aos 60 dias após a semeadura das espécies vegetais de cobertura vegetal do solo, os genótipos de milho foram semeados sob a palhada na densidade de 10 plantas por metro linear, com objetivo de população final de 200 mil plantas por hectare até o estádio vegetativo V3 da cultura. 
Tabela 1. Tabela com os valores de plantas por metro linear e por hectare, para as plantas de coberturas.

\begin{tabular}{lll}
\hline Plantas de Cobertura & Plantas por m linear & Plantas por hectare \\
\hline Brachiaria ruziziensis & 15 & $300 \mathrm{mil}$ \\
Milheto (Pennisetum glaucum (L.)) & 15 & $300 \mathrm{mil}$ \\
Feijão guandu (Cajanus cajan) & 10 & $200 \mathrm{mil}$ \\
Crotalária espectabilis & 10 & $200 \mathrm{mil}$ \\
Crotalária juncea & 10 & $200 \mathrm{mil}$ \\
\hline
\end{tabular}

Fonte: Autores.

Para determinação dos atributos químicos do solo, foram coletadas amostras de solo nas camadas de 0-20 cm de profundidade. Por tratamento foram coletadas 4 amostras simples para compor uma amostra composta de solo, nos dois anos experimentais. Foram realizadas análises de macro e micronutrientes.

Antes de cada plantio realizou-se adubação com $60 \mathrm{~kg} \mathrm{~N}$ ha $^{-1}$ via ureia $(45 \% \mathrm{~N})$, parcelada em $50 \%$ na fundação e $50 \%$ em cobertura 30 dias após semeio, $40 \mathrm{~kg} \mathrm{KCl} \mathrm{ha}^{-1}$ via cloreto de potássio $(48 \% \mathrm{KCl})$ e $60 \mathrm{~kg} \mathrm{P}_{2} \mathrm{O}_{5}$ ha $^{-1}$ via superfosfato simples (18\% de $\mathrm{P}_{2} \mathrm{O}_{5}$ ), seguindo recomendação para os solos da região (IPA, 2008).

\section{4 Índice de refletância}

A aferição foi realizada por drone, Veículo Aéreo Não Tripulado (VANT). As fotos foram tiradas com overlap e sidelap de $70 \%$ para melhor precisão e detalhamento do ortomosaico.

A altura de vôo foi de $20 \mathrm{~m}$ com GSD (Ground sample distance) de 0,03 m. O modelo dos VANTs utilizados foram o Phantom 4 (DJI ${ }^{\circledR}$ ) para a safra 2018/2019 e o FIMI A3 (Xiaomi ${ }^{\circledR}$ ) para safra 2019/2020. As imagens foram feitas no estádio fenológico de floração do milho, na qual o milho tem sua altura estabilizada. Os voos foram realizados aos 90 dias após plantio, sempre no período das $12 \mathrm{~h}$ do dia 15 de agosto em ambas as safras.

A metodologia dos índices consiste na leitura das bandas RGB (vermelho, verde e azul) de reflexão da folha, tendo estas submetidas a equações já consolidadas (Quadro 1), para gerar valores que correspondem à saúde da cultura estudada. Estes índices foram calculados via programa QGIS versão 3.10.11 através de cálculos raster. 
Quadro 1. Lista dos índices vegetais que foram utilizados.

\begin{tabular}{|c|c|c|c|}
\hline Nome do Índice & Sigla & Fórmula & Autores \\
\hline Redness Index & RI & $(\mathrm{r}-\mathrm{g}) /(\mathrm{r}+\mathrm{g})$ & Huete \& Escadafal (1991) \\
\hline Excess Green Index & ExG & $(2 \times \mathrm{g})-\mathrm{r}-\mathrm{b} /(\mathrm{r}+\mathrm{g}+\mathrm{b})$ & Meyer et al. (1998) \\
\hline $\begin{array}{c}\text { Excess Red Vegetative } \\
\text { Index }\end{array}$ & ExR & $(1.4 \mathrm{x} \mathrm{r})-\mathrm{g} /(\mathrm{r}+\mathrm{g}+\mathrm{b})$ & Louhaichi et al. (2001) \\
\hline $\begin{array}{c}\text { Ground Level Image } \\
\text { Analysis }\end{array}$ & GLI & $(\mathrm{g}-\mathrm{r})+(\mathrm{g}-\mathrm{b})) /(\mathrm{r}+\mathrm{g}+\mathrm{b})$ & Gitelson et al. (2002) \\
\hline $\begin{array}{c}\text { Visible Atmospherically } \\
\text { Resistant Index }\end{array}$ & VARIgreen & $(\mathrm{Rr}) /(\mathrm{Rg}+\mathrm{Rr}-\mathrm{Rb})$ & Kataoka et al. (2003) \\
\hline $\begin{array}{c}\text { Color Index of } \\
\text { Vegetation Extraction }\end{array}$ & CIVE & $(0,441 * \mathrm{r})-\left(0,811^{*} \mathrm{~g}\right)+(0,385 * \mathrm{~b})+18,78745$ & Hunt Júnior et al. (2005) \\
\hline $\begin{array}{c}\text { Normalized green-Red } \\
\text { Difference Index }\end{array}$ & NGRDI & $(\mathrm{g}-\mathrm{r}) /(\mathrm{g}+\mathrm{r})$ & Meyer \& Camargo Neto \\
\hline $\begin{array}{c}\text { Excess Red-Green } \\
\text { (2008) }\end{array}$
\end{tabular}

Fonte: Autores.

\subsection{Análise estatística}

Os dados foram submetidos à análise de variância (ANOVA) pelo teste $\mathrm{F}$ e em seguida os valores médios foram comparados pelo teste de Tukey $(\mathrm{p} \leq 0,05)$. Todas as análises foram realizadas com auxílio do programa $\mathrm{R}(\mathrm{R}$ Core Team).

\section{Resultados}

Para a interação genótipos de milho e sistemas de cultivo não houve significância segundo o teste $\mathrm{F}$ em ambas as safras estudadas (Tabela 1), portanto os fatores estudados não possuem dependência na influência dos índices estudados.

Entretanto, isoladamente os genótipos de milho diferiram significativamente $(\mathrm{p} \leq 0,01)$ pelo teste $\mathrm{F}$ para os índices Color Index of Vegetation Extraction (CIVE), Excess Red Vegetative Index (ExR), Excess Red-Green (ExRG), Ground Level Image Analysis (GLI), Normalized green-Red Difference Index (NGRDI) na safra 2018/2019 e Color Index of Vegetation Extraction (CIVE), Excess Green Index (ExG), Excess Red Vegetative Index (ExR), Excess Red-Green (ExRG), Ground Level Image Analysis (GLI) e o Visible Atmospherically Resistant Index (VARIgreen) para safra 2019/2020 (Tabela 1).

Em relação aos sistemas de cultivo, na primeira safra não houve efeito significativo nos índices estudados (Tabela 1), porém na segunda safra teve diferença significativa $(\mathrm{p} \leq 0,01)$ para os índices Excess Green Index (ExG), Excess Red-Green (ExRG), Ground Level Image Analysis (GLI) e Normalized green-Red Difference Index (NGRDI). 
Tabela 1. Análise de variância para os índices Color Index of Vegetation Extraction (CIVE), Excess Green Index (ExG), Excess Red Vegetative Index (ExR), Excess Red-Green (ExRG), Ground Level Image Analysis (GLI), Normalized green-Red Difference Index (NGRDI), Redness Index (RI) e o Visible Atmospherically Resistant Index (VARIgreen) para as safras 2018/2019 e 2019/2020.

\begin{tabular}{|c|c|c|c|c|c|c|c|c|}
\hline \multicolumn{9}{|c|}{$2018 / 2019$} \\
\hline FV & CIVE & ExG & ExR & ExRG & GLI & NGRDI & RI & VARIgreen \\
\hline Genótipos (G) & $0,0132 * *$ & $0,0571 \mathrm{~ns}$ & $0,0410 * *$ & $0,00344 * *$ & $0,0000 * *$ & $0,0000 * *$ & $0,86436 \mathrm{~ns}$ & $0,49008 \mathrm{~ns}$ \\
\hline Cultivo (C) & $0,7611 \mathrm{~ns}$ & $0,4338 \mathrm{~ns}$ & $0,8930 \mathrm{~ns}$ & $0,71911 \mathrm{~ns}$ & $0,3965 \mathrm{~ns}$ & $0,3381 \mathrm{~ns}$ & $0,3815 \mathrm{~ns}$ & $0,66721 \mathrm{~ns}$ \\
\hline $\mathrm{G}^{*} \mathrm{C}$ & $0,19097 \mathrm{~ns}$ & $0,4616 \mathrm{~ns}$ & $0,31691 \mathrm{~ns}$ & $0,8388 \mathrm{~ns}$ & $0,4273 \mathrm{~ns}$ & $0,31427 \mathrm{~ns}$ & $0,5158 \mathrm{~ns}$ & $0,47096 \mathrm{~ns}$ \\
\hline CV (\%) & 40,28 & 8,83 & 13,05 & 19,66 & 18,21 & 19,66 & 41,93 & 24,03 \\
\hline \multicolumn{9}{|c|}{$2019 / 2020$} \\
\hline FV & CIVE & ExG & ExR & ExRG & GLI & NGRDI & RI & VARIgreen \\
\hline Genótipos (G) & $0,00053 * *$ & $0,00007 * *$ & $0,00018 * *$ & $0,02111 * *$ & $0,02101 * *$ & $0,13787 \mathrm{~ns}$ & $0,41114 \mathrm{~ns}$ & $0,0000 * *$ \\
\hline Cultivo (C) & $0,94063 \mathrm{~ns}$ & $0,00153 * *$ & $0,29386 \mathrm{~ns}$ & $0,04977 * *$ & $0,02402 * *$ & $0,00037 * *$ & $0,16932 \mathrm{~ns}$ & $0,19778 \mathrm{~ns}$ \\
\hline $\mathrm{G}^{*} \mathrm{C}$ & $0,50784 \mathrm{~ns}$ & $0,33652 \mathrm{~ns}$ & $0,53516 \mathrm{~ns}$ & $0,95154 \mathrm{~ns}$ & $0,74097 \mathrm{~ns}$ & $0,53143 \mathrm{~ns}$ & $0,74503 \mathrm{~ns}$ & $0,8983 \mathrm{~ns}$ \\
\hline CV (\%) & 9,71 & 7,36 & 5,58 & 9,67 & 15,59 & 18,73 & 37,25 & 39,94 \\
\hline
\end{tabular}

** Significativo a $1 \%$ de probabilidade segundo teste F. FV: fonte de variação. Fonte: Autores.

Observa-se que os índices RI, ExG e VARIgreen não são indicados para observar diferenças entre genótipos de milho segundo as condições deste experimento na safra 2018/2019 (Figura 2). 
Figura 2. Imagem aérea geoprocessada, expondo os índices Visible Atmospherically Resistant Index (VARIgreen), Redness Index (RI), Normalized green-Red Difference Index (NGRDI), Ground Level Image Analysis (GLI), Excess Red-Green (ExRG), Excess Red Vegetative Index (ExR), Excess Green Index (ExG) e o Color Index of Vegetation Extraction (CIVE) para a safra 2018/2019 de milho do mês de agosto.
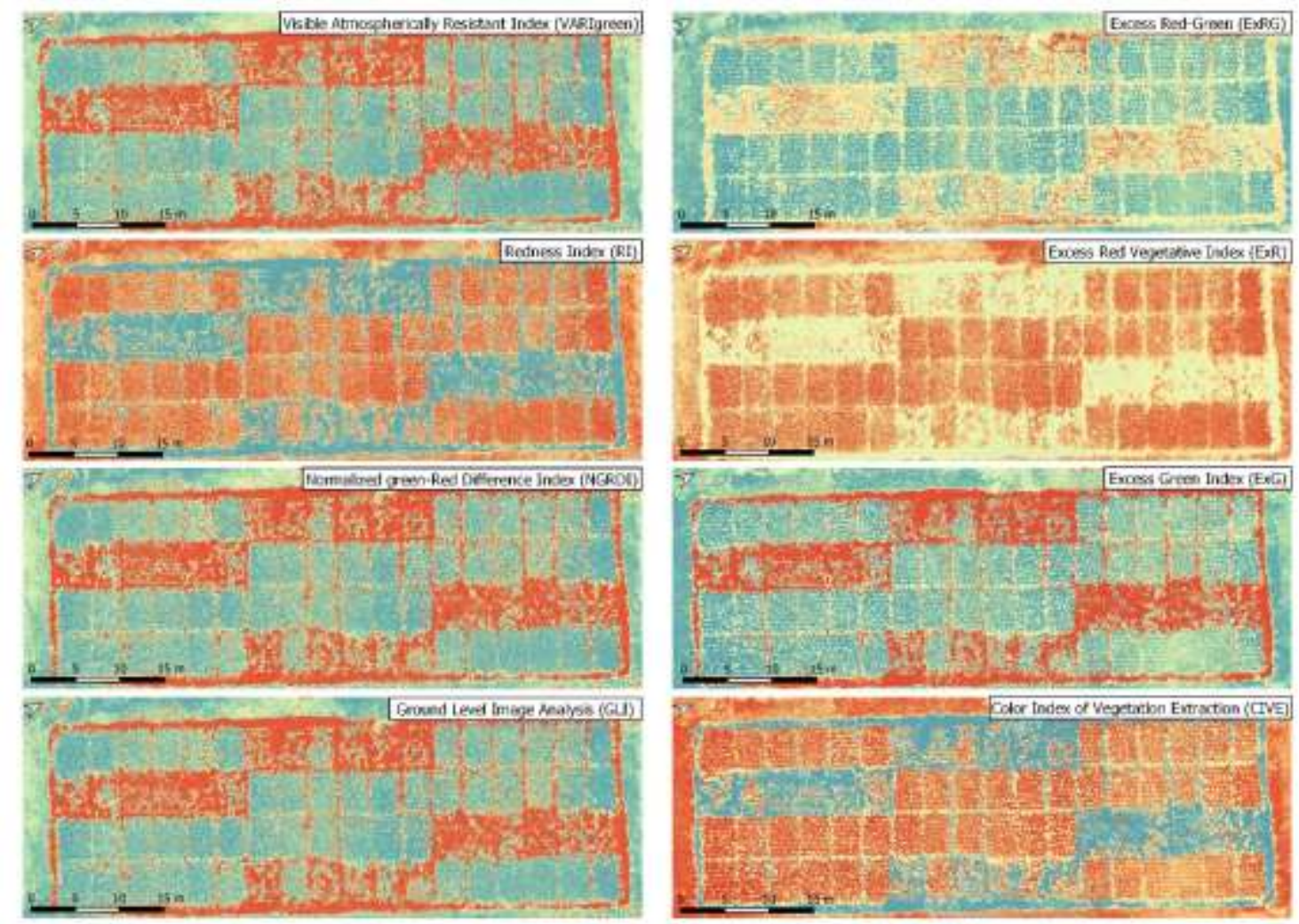

Fonte: Autores.

Para a safra 2019/2020 (Figura 3) apenas os índices Normalized Green-Red Diference Index (NGRDI) e Redness Index (RI) não apresentaram diferenças para o teste F. Quando avaliam-se as duas safras, o único índice que se mantém com mesmo padrão nos dois anos é o índice Excess Red Vegetative Index (ExR) (Figuras 2 e 3). 
Figura 3. Imagem aérea geoprocessada, expondo os índices Visible Atmospherically Resistant Index (VARIgreen), Redness Index (RI), Normalized green-Red Difference Index (NGRDI), Ground Level Image Analysis (GLI), Excess Red-Green (ExRG), Excess Red Vegetative Index (ExR), Excess Green Index (ExG) e o Color Index of Vegetation Extraction (CIVE) para a safra 2018/2019 de milho no mês de agosto.
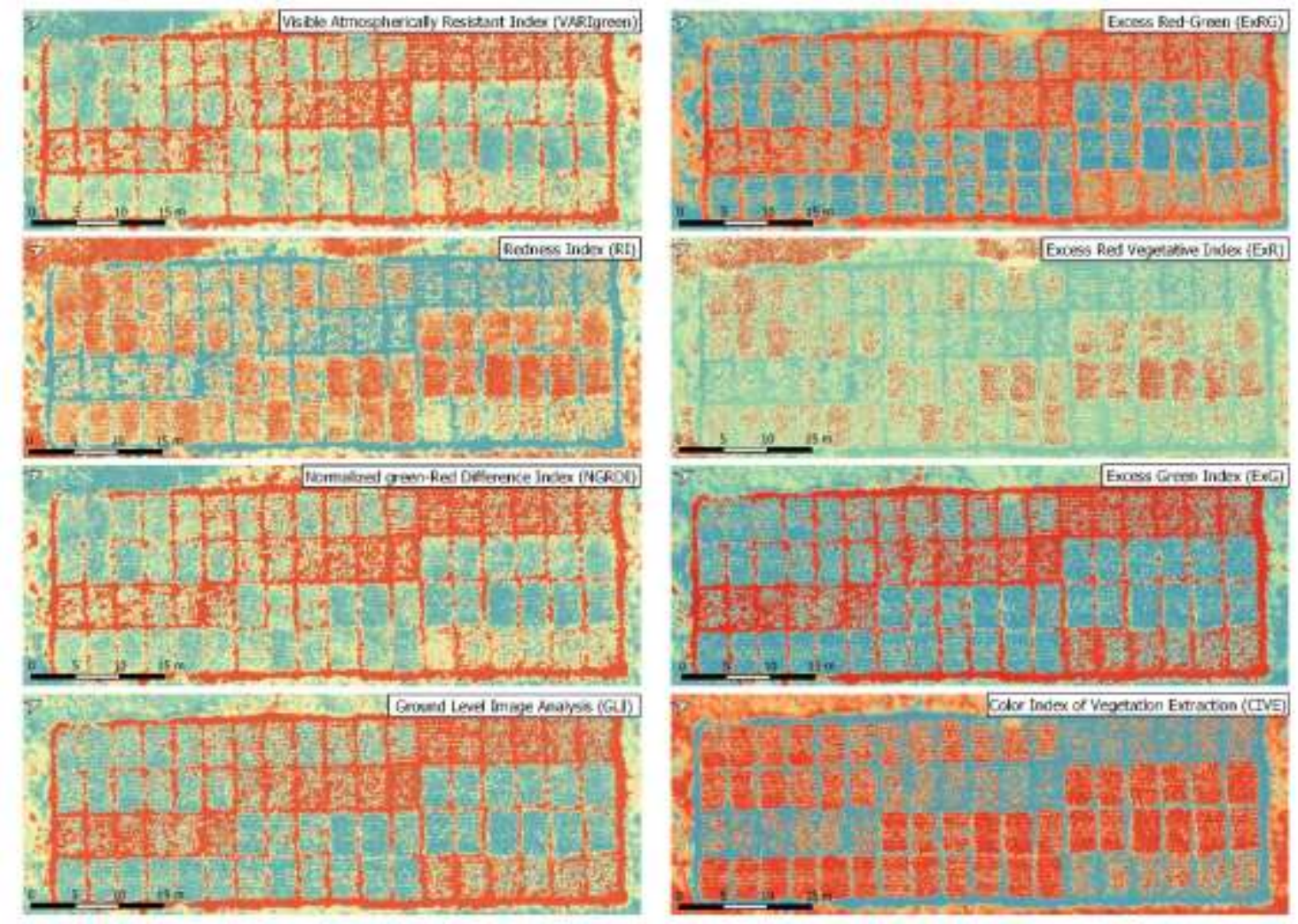

Fonte: Autores.

De acordo com Tabela 2, na safra 2018/2019 nos índices CIVE e ExR o genótipo de milho Robusto foi superior 27,99\% e 20,32\%, respectivamente, ao AG 1051. Enquanto que nos índices ExRG, GLI e NGRDI os genótipos AG 1051 e Pontinha apresentaram maiores valores e diferiram estatisticamente do genótipo Robusto, com inferioridade de 16,48 (ExRG), 32,02 (GLI) e 29,06 (NGRDI) em relação aos demais genótipos.

Na safra 2019/2020 (Tabela 2) os índices CIVE e VARIgreen apresentaram maiores valores com o genótipo AG 1051, diferindo estatisticamente dos demais genótipos. Ao tempo que os índices ExG, ExR, ExRG e GLI o genótipo Robusto foi superior ao genótipo AG 1051, com incrementos de 7,34\% (ExG), 6,50\% (ExR), 7,27\% (ExRG) e 11,76\% (GLI). 
Tabela 2. Médias para os genótipos estudados para os índices Color Index of Vegetation Extraction (CIVE), Excess Green Index (ExG), Excess Red Vegetative Index (ExR), Excess Red-Green (ExRG), Ground Level Image Analysis (GLI), Normalized green-Red Difference Index (NGRDI), Redness Index (RI) e o Visible Atmospherically Resistant Index (VARIgreen) para as safras 2018/2019 e 2019/2020.

\begin{tabular}{|c|c|c|c|c|c|c|c|c|}
\hline \multicolumn{9}{|c|}{$2018 / 2019$} \\
\hline Genótipos & CIVE & ExG & ExR & ExRG & GLI & NGRDI & RI & VARIgreen \\
\hline AG 1051 & $52,69 \mathrm{~b}$ & $507,73 a$ & $295,29 \mathrm{~b}$ & $61756,52 \mathrm{a}$ & $0,79 \mathrm{a}$ & 0,89 a & $0,19 a$ & $5,84 \mathrm{a}$ \\
\hline Pontinha & $56,70 \mathrm{ab}$ & $535,96 a$ & $315,05 \mathrm{ab}$ & 62904,48 a & $0,74 \mathrm{a}$ & $0,83 \mathrm{a}$ & $0,19 a$ & $9,88 \mathrm{a}$ \\
\hline Robusto & $73,18 \mathrm{a}$ & $508,01 \mathrm{a}$ & 325,45 a & $52055,35 \mathrm{~b}$ & $0,52 \mathrm{~b}$ & $0,61 \mathrm{~b}$ & $0,18 \mathrm{a}$ & $4,48 \mathrm{a}$ \\
\hline \multicolumn{9}{|c|}{$2019 / 2020$} \\
\hline Genótipos & CIVE & ExG & ExR & ExRG & GLI & NGRDI & RI & VARIgreen \\
\hline AG 1051 & $41,11 \mathrm{a}$ & $154,04 \mathrm{~b}$ & $104,89 \mathrm{~b}$ & $143,17 \mathrm{~b}$ & $0,75 \mathrm{~b}$ & $0,74 \mathrm{a}$ & $0,29 \mathrm{a}$ & $11,21 \mathrm{a}$ \\
\hline Pontinha & $36,66 \mathrm{~b}$ & $170,04 \mathrm{a}$ & $111,38 \mathrm{a}$ & $152,67 \mathrm{ab}$ & $0,78 \mathrm{ab}$ & $0,73 a$ & $0,33 \mathrm{a}$ & $3,13 \mathrm{c}$ \\
\hline Robusto & $38,10 b$ & $166,25 \mathrm{a}$ & $112,19 \mathrm{a}$ & $154,41 \mathrm{a}$ & $0,85 \mathrm{a}$ & $0,81 \mathrm{a}$ & $0,33 \mathrm{a}$ & $8,27 \mathrm{~b}$ \\
\hline
\end{tabular}

Médias seguidas pela mesma letra minúscula na coluna não diferem entre si. Fonte: Autores.

Analisando isoladamente os sistemas de cultivo (Tabela 3), na safra 2018/2019 não teve diferença estatística nos índices avaliados, porém no segundo ano, safra 2019/2020, o índice ExG foi maior nos sistemas de cultivos com uso das coberturas Crotalaria espectabilis, Milheto e sem cobertura vegetal, diferindo estatisticamente da Brachiaria ruziziensis, que foi inferior. Em relação ao índice NGRDI o sistema de cultivo com uso de cobertura vegetal Crotalaria Juncea obteve maior índice e diferiu do milho cultivado sem cobertura vegetal (testemunha) e com uso da cobertura Brachiaria ruziziensis.

Tabela 3. Médias para as plantas de coberturas estudadas para os índices Redness Index (RI), Excess Green Index (ExG), Excess Red Vegetative Index (ExR), Excess Red-Green (ExRG), Ground Level Image Analysis (GLI), Visible Atmospherically Resistant Index (VARIgreen), Normalized green-Red Difference Index (NGRDI) e o Color Index of Vegetation Extraction (CIVE) para as safras 2018/2019 e 2019/2020.

\begin{tabular}{ccccccccc}
\hline & & & \multicolumn{7}{c}{$2018 / 2019$} \\
\hline Plantas de cobertura & CIVE & ExG & ExR & ExRG & GLI & NGRDI & RI & VARIgreen \\
\hline Brachiaria ruziziensis & $57,96 \mathrm{a}$ & $502,66 \mathrm{a}$ & $305,29 \mathrm{a}$ & $571,48 \mathrm{a}$ & $0,69 \mathrm{a}$ & $0,79 \mathrm{a}$ & $0,21 \mathrm{a}$ & $3,66 \mathrm{a}$ \\
C. juncea & $62,14 \mathrm{a}$ & $506,73 \mathrm{a}$ & $310,56 \mathrm{a}$ & $560,96 \mathrm{a}$ & $0,71 \mathrm{a}$ & $0,78 \mathrm{a}$ & $0,17 \mathrm{a}$ & $7,57 \mathrm{a}$ \\
C. espectabilis & $54,33 \mathrm{a}$ & $523,34 \mathrm{a}$ & $304,04 \mathrm{a}$ & $613,12 \mathrm{a}$ & $0,73 \mathrm{a}$ & $0,86 \mathrm{a}$ & $0,18 \mathrm{a}$ & $3,98 \mathrm{a}$ \\
Feijão guandu & $64,13 \mathrm{a}$ & $507,93 \mathrm{a}$ & $312,29 \mathrm{a}$ & $623,18 \mathrm{a}$ & $0,69 \mathrm{a}$ & $0,77 \mathrm{a}$ & $0,19 \mathrm{a}$ & $13,24 \mathrm{a}$ \\
Milheto & $58,06 \mathrm{a}$ & $533,89 \mathrm{a}$ & $322,13 \mathrm{a}$ & $570,70 \mathrm{a}$ & $0,66 \mathrm{a}$ & $0,74 \mathrm{a}$ & $0,22 \mathrm{a}$ & $8,12 \mathrm{a}$ \\
Testemunha & $68,51 \mathrm{a}$ & $528,87 \mathrm{a}$ & $316,67 \mathrm{a}$ & $594,85 \mathrm{a}$ & $0,62 \mathrm{a}$ & $0,72 \mathrm{a}$ & $0,16 \mathrm{a}$ & $3,85 \mathrm{a}$ \\
\hline & & & & $2019 / 2020$ & & & \\
\hline Plantas de cobertura & CIVE & ExG & ExR & ExRG & GLI & NGRDI & RI & VARIgreen \\
\hline Brachiaria ruziziensis & $38,44 \mathrm{a}$ & $152,42 \mathrm{~b}$ & $110,85 \mathrm{a}$ & $139,03 \mathrm{a}$ & $0,74 \mathrm{a}$ & $0,68 \mathrm{bc}$ & $0,32 \mathrm{a}$ & $7,15 \mathrm{a}$ \\
C. juncea & $37,89 \mathrm{a}$ & $162,58 \mathrm{ab}$ & $108,77 \mathrm{a}$ & $156,12 \mathrm{a}$ & $0,88 \mathrm{a}$ & $0,90 \mathrm{a}$ & $0,32 \mathrm{a}$ & $9,11 \mathrm{a}$ \\
C. espectabilis & $39,36 \mathrm{a}$ & $171,75 \mathrm{a}$ & $111,13 \mathrm{a}$ & $155,85 \mathrm{a}$ & $0,84 \mathrm{a}$ & $0,83 \mathrm{ab}$ & $0,3 \mathrm{a}$ & $6,05 \mathrm{a}$ \\
Feijão guandu & $39,12 \mathrm{a}$ & $157,25 \mathrm{ab}$ & $108,05 \mathrm{a}$ & $149,77 \mathrm{a}$ & $0,77 \mathrm{a}$ & $0,75 \mathrm{abc}$ & $0,33 \mathrm{a}$ & $8,40 \mathrm{a}$ \\
Milheto & $38,35 \mathrm{a}$ & $168,5 \mathrm{a}$ & $111,53 \mathrm{a}$ & $152,92 \mathrm{a}$ & $0,81 \mathrm{a}$ & $0,79 \mathrm{abc}$ & $0,38 \mathrm{a}$ & $7,23 \mathrm{a}$ \\
Testemunha & $38,59 \mathrm{a}$ & $168,17 \mathrm{a}$ & $106,6 \mathrm{a}$ & $146,82 \mathrm{a}$ & $0,73 \mathrm{a}$ & $0,64 \mathrm{c}$ & $0,25 \mathrm{a}$ & $7,27 \mathrm{a}$ \\
\hline
\end{tabular}

Médias seguidas pela mesma letra minúscula na coluna não diferem entre si. Fonte: Autores. 


\section{Discussão}

Os resultados deste estudo indicaram que o sensoriamento remoto é uma importante ferramenta para o mapeamento da variabilidade espacial de cultivos de milho em sistemas de cultivo com plantas de cobertura vegetal do solo.

As principais vantagens de aplicação do índice ExR são a facilidade de cálculo, extração de pixels verdes, mesmo se baseando principalmente no componente vermelho e a segmentação de textura do solo (Meyer et al, 1998). Cruz (2020) observou que o ExR apresentou um bom resultado, separando as classes mais densas de cana-de-açúcar (pixels mais escuros) das menos densas de cana-de-açúcar (pixels mais claros). Assim pode-se observar que o Excess Red Vegetative Index (ExR) pode identificar principalmente a densidade de pixels escuros dos genótipos, demonstrando sua diferença por meio estatístico.

Esta informação pode ser corroborada por Hunt Júnior et al. (2005), que afirmam que o NGRDI possui uma correlação linear para valores de biomassa abaixo de $120 \mathrm{~g} \mathrm{~m}^{-2}$, possivelmente pelo fato das plantas de cobertura afetarem a biomassa do milho cultivado em sucessão.

Para Mendoza-Tafolla et al. (2021) o Normalized Green-Red Difference Index (NGRDI) teve o maior coeficiente de correlação com o valores de clorofila $(r=0,95)$ e nitrogênio $(N)(r=0,93)$. Além disso, o índice de Excess Green Index $($ ExG) obteve a maior correlação com área foliar $(r=0,92)$.

Esses índices destacam a resposta espectral nas regiões verde e vermelha e são mais precisos do que os índices usando a faixa azul. Demonstrando que ambos os índices supracitados possuem alta correlação com parâmetros fisiológicos, deste modo, pode-se deduzir que as plantas de cobertura influenciaram nestes fatores e que eles conseguiram captar essas diferenças.

O NGRDI indica o seu potencial para determinar a biomassa das culturas, estabelecendo mapas de variação de rendimento de todo um campo, além disso, índices que não usam a banda do infravermelho (infrared - IR) não conseguiram detectar diferenças de manejo em culturas como a ervilha e aveia (Jannoura et al., 2015). Porém, neste trabalho como o manejo usado afeta diretamente na nutrição e consequentemente na fotossíntese das plantas, foi possível averiguar diferença entre as coberturas usadas.

O desenvolvimento de Veículos Aéreos Não Tripulados (VANTs), como uma ferramenta para agricultura de precisão específica para cada local pode ser uma alternativa de baixo custo para métodos destrutivos, e pode ser parte de um sistema combinado com medidores portáteis (Jannoura et al., 2015; Mendoza-Tafolla et al., 2021).

\section{Considerações Finais}

Os índices estudados mostraram-se sensíveis para observar variações entre os genótipos de milho e plantas de cobertura vegetal do solo; para os genótipos de milho é indicado o uso do índice Excess Red Vegetative Index (ExR); para as plantas de cobertura do solo estudadas indica-se o Normalized green-Red Difference Index (NGRDI).

A pesquisa fornece informações importantes sobre o sensoriamento remoto nas condições do Brejo da Paraíba. Essas informações podem ajudar produtores rurais e pesquisadores para futuros trabalhos científicos, os quais auxiliarão como ferramentas do manejo agrícola aplicado à agricultura de precisão.

\section{Agradecimentos}

Os autores agradecem ao Conselho Nacional de Desenvolvimento Científico e Tecnológico (CNPq) e ao Centro de Ciências Agrárias (Campus II) da Universidade Federal da Paraíba pelo suporte financeiro.

\section{Referências}

Acco, M. (2004). Modelagem de dados do satélite Ikonos II para estimativa de micronutrientes na floresta ombrófila mista montana. Dissertação (Mestrado) Universidade Federal do Paraná, Centro de Ciências Agrárias, Curitiba. 
Alvares, C. A., Stape, J. L., Sentelhas, P. C., Gonçalves, J. L. M., \& Sparovek, (2013). Köppen's climate classification map for Brazil. Meteorologische Zeitschrift, 22, 721-728. https://doi.org/10.1127/0941-2948/2013/0507

Backes, K. S. (2010). Variações do índice de vegetação por diferença normalizada (NDVI) do sensor modis associadas a variáveis climáticas para o estado do Rio Grande do Sul. Dissertação (Mestrado) - Universidade Federal de Santa Maria. Santa Maria.

Cruz, A. C. (2020). Estimativa de densidade de plantação de cana de açúcar utilizando o espaço de cor cie lab em imagens de alta resolução espacial provindas de VANTS. Dissertação (Mestrado) - Universidade Estadual Paulista (Unesp), Instituto de Ciência e Tecnologia, Sorocaba.

Embrapa - Empresa Brasileira de Pesquisa Agropecuária (2006). Sistema brasileiro de classificação de solos. Rio de Janeiro: Embrapa, 2. ed.

Gerhardt, T. E., \& Silveira, D. T. (2009). Métodos de pesquisa. Editora da UFRGS, Brasil.

Gitelson, A. A., Kaufman, Y. J., Stark, R., \& Rundquist, D. (2002). Novel algorithms for remote estimation of vegetation fraction. Remote Sensing of Environment, 80(1), 76-87.

Huete, A. R., \& Escadafal, R. (1991). Assessment of biophysical soil properties through spectral decomposition techniques. Remote Sensing of Environment. $35,149-159$.

Hunt Júnior, E. R., Cavigelli, M., Daughtry, C. S. T., Mcmurtrey III, J. E., \& Walthall, C. L. (2005). Evaluation of digital photography from model aircraft for remote sensing of crop biomass and nitrogen status. Precision Agriculture, 6, 359-378.

IPA - Empresa Pernambucana de Pesquisa Agropecuária (2008). Recomendações de adubação para o estado de Pernambuco (2ª aproximação). 2.ed. Recife: Empresa Pernambucana de Pesquisa Agropecuária. 198p.

Jannoura, R., Brinkmann, K., Uteau, D., Bruns, C., \& Joergensen, R. C. (2015). Monitoring of crop biomass using true colour aerial photographs taken from a remote controlled hexacopter. Biosystems Engineering, 129, 341-351.

Kalisch, L., Dalmas, F. B., Paranhos Filho, A. C., \& Encina, C. C. (2020). Análise da cobertura vegetal da RPPN Fazenda Uhumirim por meio de sensoriamento remoto. Research, Society and Development, 9(4): e33942737

Kataoka, T., Kaneko, T., Okamoto, H., \& Hata, S. (2001). Crop growth estimation system using machine vision. In: Proceedings 2003 IEEE/ASME International Conference on Advanced Intelligent Mechatronics (AIM 2003). IEEE, 2003. p. b1079-b1083 vol. 2.

Louhaichi, M., Borman, M. M., \& Johnson, D. E. (2001). Spatially located platform and aerial photography for documentation of grazing impacts on wheat. Geo car to International, 16(1): 65-70.

Mendoza-Tafolla, R. O., Ontiveros-Capurata, R. E., Juarez-Lopez, P., Alia-Tejacal, I., Lopez-Martinez, V., \& Ruiz-Alvarez, O. (2021). Nitrogen and chlorophyll status in romaine lettuce u sing spectral indices from RGB digital images. Zemdirbyste-Agriculture, 108(1): 79-86.

Meyer, G. E., Hindman, T., \& Laksmi, K., M. G. (ed.), Deshazer J. A., Machine vision detection parameters for plant species identification. Precision agri540 culture and biological quality, Boston, Massachusetts, USA, 34 November, 19983543 (November) (1998) 327-335

Meyer, G. E., \& Camargo Neto J., (2008). Verification of color vegetation indices for automated crop imaging applications, Computers and Electronics in Agriculture. 63(2), 282-293.

Mumbach, G. L., Kotowski, I. E., Schneider, F. J. A., Mallmann, M. S., Bonfada, E. B., Portela, V. O., Bonfada, E. B., \& Kaiser, D. R. (2017). Resposta da inoculação com Azonspirillum brasilense nas culturas de trigo e milho safrinha. Revista Scientia Agraria, 18(2), 97-103.

Pereira, J. L. A. R., Von Pinho, R. G., Souza Filho, A. X., Pereira, M. N., Santos, A. O., \& Borges, I. D (2012). Quantitative characterization of corn plant components according to planting time and grain maturity stage. Revista Brasileira de Zootecnia, 41(5), $1110-1117$.

Ribeiro, J. E. S., Barbosa, A. J. S., Lopes, S. F., Pereira, W. E., \& Albuquerque, M. B. (2018). Seasonal variation in gas exchange by plants of Erythroxylum simonis Plowman. Acta Botanica Brasílica 32, 287-296. https://doi.org/10.1590/0102- 33062017abb0240

Ribeiro, E. S., Faro, B. L. S. O., Nascimento, R. S. C., Carneiro, F. S., Amaral, A. P. M., Bezerra, M. G. S., Amorim, M. B., \& Garcia, T. S. (2021). Multitemporal evaluation of the vegetation cover of the Tapirapé biological reserve, Pará. Research, Society and Development, 10(4): e50910414345

Woebbecke, D. M., Meyer, G. E., Von Bargen, K., \& Montensen, D. A. (1995). Color indices for weed identification under various soil, residue, and lighting conditions. Transactions of the ASAE, 38(1), 259-269. 